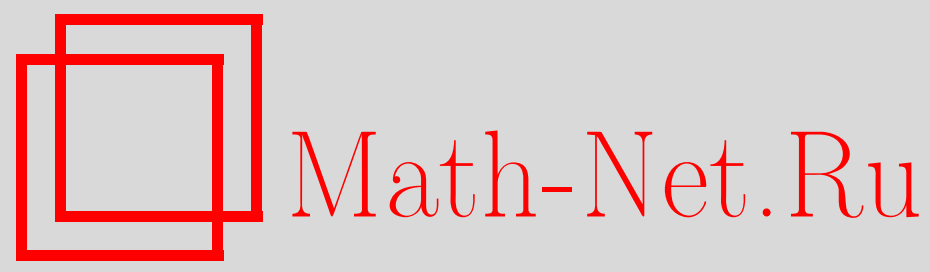

В. Е. Тараканов, Об одном применении леммы Гаусса при изучении псевдослучайных последовательностей, основанных на квадратичных вычетах, Матем. заметки, 2003, том 73, выпуск 4, 603-612

DOI: https://doi.org/10.4213/mzm208

Использование Общероссийского математического портала Math-Net.Ru подразумевает, что вы прочитали и согласны с пользовательским соглашением http://www.mathnet.ru/rus/agreement

Параметры загрузки:

IP: 34.239 .49 .27

26 апреля 2023 г., 18:05:27

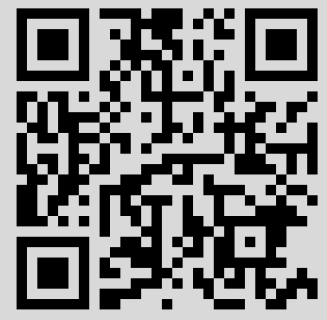




\title{
ОБ ОДНОМ ПРИМЕНЕНИИ ЛЕММЫ ГАУССА ПРИ ИЗУЧЕНИИ ПСЕВДОСЛУЧАЙНЫХ ПОСЛЕДОВАТЕЛЬНОСТЕЙ, ОСНОВАННЫХ НА КВАДРАТИЧНЫХ ВЫЧЕТАХ
}

\author{
В. Е. Тараканов
}

\begin{abstract}
В связи с изучением псевдослучайных последовательностей, использующих квадратичные вычеты по модулю простого числа $p$, рассматривается задача о конструктивном описании множества простых модулей, для которых заданные целые числа являются квадратичными вычетами. На основе леммы Гаусса устанавливается критерий комбинаторного характера для того, чтобы данное целое число $a$ являлось квадратичньп вычетом по модулю простого числа $p$. Показано, как этот критерий может быть применен к задаче об эффективном описании простых модулей $p$ со свойством $\left(\frac{a}{p}\right)=1$ для каждого $p$ из заданного конечного множества $M$.
\end{abstract}

Библиография: 6 названий.

Квадратичные вычеты по модулю простого числа $p$ или, вообще, числа с равным 1 значением символа Якоби по модулю нечетного числа $m$ применяются в ряде современных способов построения псевдослучайных последовательностей (см. [1]-[4]). При изучении таких последовательностей возникает задача описания в явном виде множества всех нечетных чисел $m$, принадлежащих заданному множеству $M$, для которых каждое из целых чисел некоторой совокупности $\left\{a_{1}, \ldots, a_{l}\right\}$ имеет значение символа Якоби, равное 1:

$$
\left(\frac{a_{i}}{m}\right)=1 \quad \text { для каждого } m \in M, i=1, \ldots, l .
$$

Мы рассматриваем в работе один подход к решению этой задачи. Для этого получим вначале некоторый комбинаторньй критерий того, что данное целое число $a>0$ является квадратичным вычетом по модулю простого числа $p>a$, основанный на известной лемме Гаусса. Затем, основываясь на нем, мы укажем возможный способ конструктивного описания тех модулей $m$ из $M$, которые являются простьми числами и удовлетворяют условию $\left(\frac{a}{m}\right)=1, m \in M$, для данного целого $a, 0<a<m$.

Используемые факты и понятия из теории чисел можно найти в книгах [5], [6]. Для нечетного целого $m$ и целого числа $a$, взаимно простого $c m$, через $\left(\frac{a}{m}\right)$ обозначаем символ Якоби $a$ по модулю $m$. Отправной точкой для последующего служит следующий классический результат.

Работа выполнена при поддержке программы "Ведущие научные школы" Российского фонда фундаментальных исследований, грант № 00-15-96-136. 


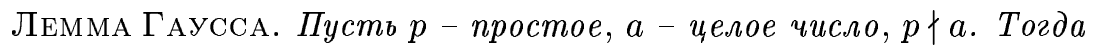

$$
\left(\frac{a}{p}\right)=(-1)^{n},
$$

әде $n$ есть количество отрицательных вычетов среди абсолютно наименьших вычетов $(\bmod p)$ для кратных $1 \cdot p, 2 \cdot p, \ldots,(p-1) / 2 \cdot p$.

Выведем из условия, выражаемого формулой (1), критерий для квадратичных вычетов $(\bmod p)$, зависящий не от $p$, а от класса вычетов $(\bmod a)$, которому принадлежит $p$. Согласно теореме Дирихле о простых числах этот класс содержит бесконечно много простых чисел.

Введем предварительно несколько определений и сделаем несколько очевидных замечаний.

ОПРЕДЕЛЕНИЕ. Пусть $m, a-$ два положительных целых числа, $t=\max \{k: k-$ целое, $k a<m\}$. Соотношение

$$
m=t a+n_{1}, \quad n_{1} \leqslant a,
$$

назовем стандартным представлением $m$ через $a$.

Ясно, что

$$
t= \begin{cases}{\left[\frac{m}{a}\right],} & a \nmid m, \\ \frac{m}{a}-1, & a \mid m .\end{cases}
$$

Для нечетного целого $m$ и целого $a, 0<a<m$, взаимно простого $c m$, часто будем рассматривать вместе с (1) также стандартное представление

$$
\frac{m+1}{2}=s a+n_{2}, \quad n_{2} \leqslant a,
$$

$(m+1) / 2$ через $a$.

ЗАмЕчАнИЕ 1 . Пусть $m$ - нечетное, $a$ - целое число, $0<a<m, \operatorname{HOД}(m, a)=1$, $(2)$ и $(3)$ - стандартные представления $m$ и $(m+1) / 2$ через $a$. Тогда

$$
\begin{gathered}
n_{2}= \begin{cases}\frac{n_{1}+1}{2}, & \text { если } t \text { четно, } \\
\frac{n_{1}+1+a}{2}, & \text { если } t \text { нечетно, }\end{cases} \\
s= \begin{cases}\frac{t}{2}, \quad \text { если } t \text { четно, } \\
\frac{t-1}{2}, \text { если } t \text { нечетно. }\end{cases}
\end{gathered}
$$

Действительно, пусть $t$ нечетно. Тогда $t=2 t_{1}+1, t_{1}=(t-1) / 2$ и $m+1=2 a t_{1}+a+$ $n_{1}+1$. Тем самьм, $a+n_{1}+1$ четно и, очевидно,

$$
\frac{m+1}{2}=a t_{1}+\frac{a+n_{1}+1}{2}
$$

- стандартное представление. Аналогично рассуждаем при четном $t$. 
ЗАмЕчАниЕ 2 . Пусть $m, a$ - целые числа, $m$ нечетно, $0<a<m$, НОД $(m, a)=1$. Тогда в (2), (3) имеем

$$
\begin{array}{ll}
n_{2} \leqslant n_{1}, & \text { если } t \text { четно, } \\
n_{1}<n_{2}, & \text { если } t \text { нечетно, }
\end{array}
$$

при этом $n_{1}=n_{2}$ равносильно $n_{1}=1$, а $n_{2}=a$ равносильно $n_{1}=a-1$.

ОПрЕДЕЛЕНИЕ. Пусть $m, a, b, k$ - целые числа, $0<|b|<a \leqslant m, k \geqslant 1$. Назовем арифметической прогрессией длины $k$ по модулю $m$ с начальным членом а и разностью $b$ последовательность

$$
P_{m}^{(k)}(a, b)=\left(a_{1}^{*}, a_{2}^{*}, \ldots, a_{k}^{*}\right),
$$

где $a_{i}^{*} \equiv a+(i-1) b(\bmod m), 0<a_{i}^{*} \leqslant m, i=1,2, \ldots, k$. При этом монотонную последовательность $\left(c_{i_{0}}, c_{i_{0}+1}, \ldots, c_{i_{0}+l-1}\right)$ прогрессии $(7)$ будем называть ее полным отрезком, если

$$
\begin{aligned}
& c_{i_{0}}-b \leqslant 0, \quad c_{i_{0}+l-1}<m \leqslant c_{i_{0}+l-1}+b \quad \text { при } b>0, \\
& c_{i_{0}}-b>m, \quad c_{i_{0}+l-1}>0 \geqslant c_{i_{0}+l-1}+b \quad \text { при } b<0 \text {. } \\
& 1 \leqslant i_{0}, l \leqslant k \text {. }
\end{aligned}
$$

Принимая это определение, можно следующим образом сформулировать лемму Гаycca.

ПРЕДЛОЖЕНИЕ 1. Пусть $p$ - простое, $a-$ челое число, $p \nmid a$. Тогда

$$
\left(\frac{a}{p}\right)=(-1)^{n}
$$

əде $n=\left|P_{p}^{((p-1) / 2)}\left(a^{*},|a|\right) \cap[(p+1) / 2, p)\right|,|X|$ обозначает мошность мнохсества $X$.

Если рассматриваем прогрессию $(7)$, не фиксируя ее длины, то пишем просто $P_{m}(a, b)$.

ЗАмЕчАнИЕ 3 . Пусть $m$ - нечетное, $a$ - целое число, $0<a<m, \mathrm{HOД}(m, a)=1, b-$ целое число, $0<b \leqslant a$. Пусть, далее,

$$
m=t a+n_{1}, \quad \frac{m+1}{2}=s a+n_{2}
$$

- стандартные представления (1) и (2). Тогда

$1)$ полный отрезок прогрессии $P_{m}(b, a)$ имеет длину $t$, если $b \geqslant n_{1}$, и длину $t+1$, если $0<b<n_{1}$;

$2)$ полный отрезок прогрессии $P_{(m+1) / 2}(b, a)$ имеет при четном $t$ длину $t / 2$, если $b \geqslant n_{2}$, и длину $t / 2+1$, если $0<b<n_{2}$; при нечетном $t$ длину $(t-1) / 2$, если $b \geqslant n_{2}$, и длину $(t+1) / 2$, если $0<b<n_{2}$.

Утверждения следуют из замечаний 1,2 .

Зафиксируем теперь нечетное простое число $p$. Для целого $k$ обозначим $a^{(k)}$ наименьший неотрицательньй вычет $(\bmod p)$ числа $k a$ :

$$
a^{(k)} \equiv k a(\bmod p), \quad 0 \leqslant a^{(k)}<p .
$$

Для целого числа $a, 0<a \leqslant p-1$, рассмотрим последовательность $\Pi_{a}=P_{p}^{(p-1)}(a, a)$ :

$$
\Pi_{a}=\left(a^{(1)}, a^{(2)}, \ldots, a^{(p-1)}\right) .
$$

Отметим ряд очевидных свойств прогрессии $\Pi_{a}$ по модулю $p$. 
ЗАмЕчаниЕ 4. Элементы $\Pi_{a}$ образуют некоторую перестановку чисел $1,2, \ldots, p-1$.

Рассмотрим полные отрезки в последовательности П $a$. Пусть $t, n_{1}-$ числа из стандартного представления (1) для $m=p$.

ЗАмЕчАниЕ 5 . Прогрессия $\Pi_{a}$ разбивается на подряд идущие полные отрезки $\pi_{1}$, $\pi_{2}, \ldots$ :

$$
\Pi_{a}=\left(\left(\pi_{1}\right),\left(\pi_{2}\right), \ldots\right)
$$

длины $t$ или $t+1$ каждьй.

Утверждение следует из замечания 3.

ЗАмЕчАниЕ 6. Количество полных отрезков в (9) равно $а$. Начальные члены этих отрезков образуют арифметическую прогрессию $P_{a}^{(a)}\left(a,-n_{1}\right)$ по модулю $a$.

Утверждение справедливо ввиду НОД $\left(a, n_{1}\right)=1$ и замечания 4.

ЗАМЕЧАнИЕ 7. В (9) имеется $n_{1}-1$ полных отрезков длины $t+1$ и $a-n_{1}+1$ полных отрезков длины $t$.

Утверждение следует из замечаний 3,5 и 6.

Таким образом, прогрессии $\Pi_{a}$ из (8) соответствует последовательность

$$
\widetilde{\Pi}_{a}=\left(\pi_{1}, \pi_{2}, \ldots, \pi_{a}\right),
$$

где $\pi_{l}$ есть полный отрезок в (9) длины $t$ или $t+1$ с начальным членом $a$ при $l=1$ и

$$
a_{l} \equiv a_{l-1}-i_{l} n_{1}(\bmod a), \quad 0<a_{l}<a, \quad l=2,3, \ldots, a,
$$

где $i_{l}$ - минимальное целое число со свойством

$$
a_{l-1}-\left(i_{l}-1\right) n_{1}>0>a_{l-1}-i_{l} n_{1}
$$

ЗАмЕчаниЕ 8. Длина полных отрезков $\pi_{1}$ и $\pi_{a}$ в $(10)$ равна $t$.

Действительно, $t a<p<(t+1) a$, поэтому длина $\pi_{1}$ равна $t$. Кроме того,

$$
(p-f) a \equiv p-f a(\bmod p), \quad f=1,2, \ldots,
$$

и из $0<a<\cdots<t a<p$ следует, что $0<p-t a<\cdots<p-a<p$, т.е. длина $\pi_{a}$ равна также $t$.

Рассмотрим теперь первую половину последовательности $\Pi_{a}$ из (8):

$$
\Pi_{a}^{\prime}=\left(a, a^{(2)}, \ldots, a^{((p-1) / 2)}\right)
$$

ЛЕмМА. При а четном $\Pi_{a}^{\prime}$ из (11) представляется как последовательность идущих подряд а/2 полных отрезков:

$$
\Pi_{a}^{\prime}=\left(\left(\pi_{1}\right),\left(\pi_{2}\right), \ldots,\left(\pi_{a / 2}\right)\right)
$$

При а нечетном $\Pi_{a}^{\prime}$ представляется в виде

$$
\Pi_{a}^{\prime}=\left(\left(\pi_{1}\right),\left(\pi_{2}\right), \ldots,\left(\pi_{(a-1) / 2}\right),(\widetilde{\pi})\right),
$$

где $\pi_{1}, \ldots, \pi_{(a-1) / 2}$ - полные отрезки, а $\widetilde{\pi}-$ подпоследовательность $\Pi_{a}^{\prime}$ длины $t / 2$ при четном $t u(t+1) / 2$ при $t$ нечетном; если $c \in \tilde{\pi}$, то $c \leqslant(p-1) / 2$. 
ДокАЗАтЕльство. Рассмотрим последовательность (10). Пусть для некоторогоцелого $b, 0<b \leqslant a$, и некоторого $l, 1 \leqslant l \leqslant a$, имеем

$$
\pi_{l}=\left(b, b+a, \ldots, b+\left(t^{\prime}-1\right) a\right)
$$

где $\pi_{l}-$ полньй отрезок, т.е. $t^{\prime}=t$ или $t+1$ (см. замечание 5$)$. Обозначим

$$
-\pi_{l}=\left(p-\left(b+\left(t^{\prime}-1\right) a\right), p-\left(b+\left(t^{\prime}-2\right) a\right), \ldots,(p-b)\right) .
$$

Тогда ввиду полноты $\pi_{l}$ имеем

$$
0<p-\left(b+\left(t^{\prime}-1\right) a\right) \leqslant a,
$$

и ввиду $b \leqslant a$

$$
(p-b)+a \geqslant p .
$$

Поэтому последовательность $-\pi_{l}$ из (14) есть полньй отрезок, один из членов последовательности (10). Из определения $\Pi_{a}\left(\right.$ см (8)) ясно, что $a^{(p-1)}=p-a$. Поэтому, учитьвая замечание 8 , видим, что $\pi_{a}=-\pi_{1}$. Отсюда по индукции получаем, что

$$
-\pi_{l}=\pi_{a-l+1}, \quad l=1,2, \ldots, a .
$$

Тем самьм, при четном $a(10)$ можно записать в виде

$$
\widetilde{\Pi}_{a}=\left(\pi_{1}, \pi_{2}, \ldots, \pi_{a / 2},-\pi_{a / 2},-\pi_{a / 2-1}, \ldots,-\pi_{1}\right),
$$

и мы приходим к представлению (12).

При $a$ нечетном пусть $\pi_{(a+1) / 2}=\left(c_{1}, c_{2}, \ldots, c_{t^{\prime}}\right), c_{1}<c_{2}<\cdots<c_{t^{\prime}}$. Из (15) получаем $-\pi_{(a+1) / 2}=\pi_{(a+1) / 2}$. Следовательно,

$$
p-c_{i}=c_{t^{\prime}-i+1}, \quad i=1,2, \ldots, t^{\prime} .
$$

Так как $2 c \neq p$ ни для какого $c \in \pi_{(a+1) / 2}, t^{\prime}$ должно быть четным, т.е. $t^{\prime}=t$ при четном $t, t^{\prime}=t+1$ при $t$ нечетном. Ясно также, что $c_{i}<(p-1) / 2$, если $i \leqslant t^{\prime} / 2$, так как в противном случае $c_{t^{\prime}-i+1}=p-c_{i}<c_{i}$, что противоречит монотонности $\pi_{(a+1) / 2}$. Таким образом,

$$
\widetilde{\Pi}_{a}=\left(\pi_{1}, \ldots, \pi_{(a-1) / 2}, \widetilde{\pi},-\widetilde{\pi},-\pi_{(a-1) / 2}, \ldots,-\pi_{1}\right),
$$

где $\widetilde{\pi}=\left(c_{1}, c_{2}, \ldots, c_{t^{\prime} / 2}\right), t^{\prime}=t$ при четном $t$ и $t+1$ при $t$ нечетном и $c<(p-1) / 2$ для любого $c \in \widetilde{\pi}$, т.е. приходим к (13). Лемма доказана.

Таким образом, прогрессии $\Pi_{a}^{\prime}$ из (11) соответствует последовательность

$$
\widetilde{\Pi}_{a}^{\prime}= \begin{cases}\left(\pi_{1}, \pi_{2}, \ldots, \pi_{a / 2}\right), & a \text { четно, } \\ \left(\pi_{1}, \pi_{2}, \ldots, \pi_{(a-1) / 2}, \widetilde{\pi}\right), & a \text { нечетно, }\end{cases}
$$

составленная из полньх отрезков, а также из половины $\widetilde{\pi}$ полного отрезка $\pi_{(a+1) / 2}($ при нечетном $a$ ). Рассматривая для $p, a, 0<a<p$, стандартные представления $p$ и $(p+1) / 2$ через $a$, определим отрезок натурального ряда $I$ :

$$
I= \begin{cases}{\left[n_{2}, n_{1}-1\right],} & \text { если } t \text { четно, } n_{1}>1, \\ \varnothing, & \text { если } t \text { четно, } n_{1}=1, \\ {\left[n_{1}, n_{2}-1\right],} & \text { если } t \text { нечетно }\end{cases}
$$

(см. замечание 2). Определим теперь число $d$ как количество полньх отрезков в (16) с начальньми членами из $I$. 
Теорема 1. Пусть $p$ - нечетное простое, $а$-челое число, $0<a<p$,

$$
\begin{aligned}
p & =t a+n_{1}, & & n_{1}<a, \\
\frac{p+1}{2} & =s a+n_{2}, \quad & & n_{2} \leqslant a,
\end{aligned}
$$

- стандартные представления $р и(p+1) / 2$ через а. Тогда

$$
\left(\frac{a}{p}\right)= \begin{cases}(-1)^{d+1}, & \text { если одновременно } a \equiv 2,3(\bmod 4), t \equiv 1,2(\bmod 4), \\ (-1)^{d} & \text { в остальных случаях }\end{cases}
$$

ДокАЗАТЕЛЬСТво. Введем обозначения

$$
Z_{p}^{(1)}=\left\{0,1, \ldots, \frac{p-1}{2}\right\}, \quad Z_{p}^{(2)}=\left\{\frac{p+1}{2}, \frac{p+3}{2}, \ldots, p-1\right\} .
$$

Согласно предложению 1 имеем

$$
\left(\frac{a}{p}\right)=(-1)^{n}
$$

где $n=\left|\Pi_{a}^{\prime} \cap Z_{p}^{(2)}\right|$ (ясно, что при этом достаточно знать лишь четность $n$ ). Согласно лемме $n$ членов $\Pi_{a}^{\prime}$, определяюшие значение символа Лежандра, распределяются только по $[a / 2]$ полным отрезкам из $(16)$, так как $\widetilde{\pi} \subset Z_{p}^{(1)}$. Обозначаем

$$
c=\left[\frac{a}{2}\right] \text {. }
$$

Через $d_{i}$ обозначим число членов в полных отрезках $\pi_{i}$ из $(16)$, принадлежащих $Z_{p}^{(2)}$. Тогда

$$
\left(\frac{a}{p}\right)=(-1)^{\sum d_{i}}=\prod_{i=0}^{c}(-1)^{d_{i}}
$$

Ясно, однако, что к неединичному вкладу в (18) могут приводить лишь отрезки с нечетными значениями $d_{i}$. Найдем количество таких отрезков. Рассмотрим по отдельности два случая.

1) Пусть $t$ четно. Тогда $n_{2}=\left(n_{1}+1\right) / 2, n_{2} \leqslant n_{1}$ (замечания 1,2$), I=\left[n_{2}, n_{1}-1\right]$ $\left(I=\varnothing \Longleftrightarrow n_{2}=n_{1}=1\right)$. Разобьем отрезок $[1, a]$ на три части:

$$
I_{1}=\left[1, n_{2}-1\right], \quad I_{2}=I=\left[n_{2}, n_{1}-1\right], \quad I_{3}=\left[n_{1}, a\right]
$$

(среди них могут быть пустые). Пусть $\pi$ - полньй отрезок из (16) с начальным членом $b$, $1 \leqslant b \leqslant a$. Имеются три возможности.

a) Пусть $b \in I_{3}$. Так как $b \geqslant n_{1}, n_{2}$, согласно замечанию 3 число членов в $\pi$ равно $t$; из них в точности $t / 2$ принадлежат $Z_{p}^{(1)}$. Тем самым, число членов $\pi$, входящих в $Z_{p}^{(2)}$, равно $t / 2$.

б) Пусть $b \in I$. Так как $n_{2} \leqslant b<n_{1}$, согласно замечанию $3 \pi$ состоит из $t+1$ элементов, из которых в точности $t / 2$ принадлежат $Z_{p}^{(1)}$. Тем самым, число членов $\pi$, входящих в $Z_{p}^{(2)}$, равно $t / 2+1$. 
в) Пусть $b \in I_{1}$. Так как $b<n_{1}, n_{2}$, согласно замечанию $3 \pi$ состоит из $t+1$ элементов, среди которых в точности $t / 2+1$ принадлежат $Z_{p}^{(1)}$. Тем самым, число членов $\pi$, входящих в $Z_{p}^{(2)}$, равно $t / 2$.

Таким образом, при $t \equiv 0(\bmod 4)$ неединичные вклады в $(18)$ могут дать только отрезки из $(16)$ с начальньми членами из $I$, а при $t \equiv 2(\bmod 4)$ - лишь отрезки с начальньми членами из $I_{1}$ или $I_{3}$. Итак, если $t$ четно, то

$$
\left(\frac{a}{p}\right)= \begin{cases}(-1)^{d}, & t \equiv 0(\bmod 4) \\ (-1)^{c-d}, & t \equiv 2(\bmod 4)\end{cases}
$$

2) Пусть $t$ нечетно. Тогда $n_{2}=\left(n_{1}+1+a\right) / 2, n_{1}<n_{2}$ (замечания 1,2$), I=\left[n_{1}, n_{2}-1\right]$. Вновь рассмотрим разбиение $[1, a]=I_{1} \cup I_{\cup} I_{3}$, где теперь

$$
I_{1}=\left[1, n_{1}-1\right], \quad I_{2}=I=\left[n_{1}, n_{2}-1\right], \quad I_{3}=\left[n_{2}, a\right]
$$

Пусть $\pi$ - полньй отрезок из (16).

a) Пусть $b \in I_{3}$. Тогда $\pi$ состоит из $t$ членов, из которьх в точности $(t-1) / 2$ входят в $Z_{p}^{(1)}$. Следовательно, в $Z_{p}^{(2)}$ входят $(t+1) / 2$ элементов $\pi$.

б) Пусть $b \in I$. Тогда $\pi$ состоит из $t$ членов, из которых в $Z_{p}^{(1)}$ входят $(t+1) / 2$. Следовательно, в $Z_{p}^{(2)}$ входят $(t-1) / 2$ элементов $\pi$.

в) Пусть $b \in I_{1}$. Тогда $\pi$ состоит из $t+1$ членов, из которых $(t+1) / 2$ входят в $Z_{p}^{(1)}$. Следовательно, в $Z_{p}^{(2)}$ входят $(t+1) / 2$ элементов.

Поэтому при $t \equiv 3(\bmod 4)$ неединичньй вклад в $(18)$ могут внести лишь отрезки с $b \in I_{2}=I$, а при $t \equiv 1(\bmod 4)$ - лишь отрезки с $b \in I_{1} \cup I_{3}$.

Итак, если $t$ нечетно, то

$$
\left(\frac{a}{p}\right)= \begin{cases}(-1)^{d}, & t \equiv 3(\bmod 4) \\ (-1)^{c-d}, & t \equiv 1(\bmod 4)\end{cases}
$$

Из (19) и (20) получаем

$$
\left(\frac{a}{p}\right)= \begin{cases}(-1)^{d}, & t \equiv 0,3(\bmod 4) \\ (-1)^{c-d}, & t \equiv 1,2(\bmod 4)\end{cases}
$$

Если $c$ четно, т.е. $a \equiv 0,1(\bmod 4)$, то $c-d \equiv d(\bmod 2)$, а если $c$ нечетно, т.е. $a \equiv 2,3$ $(\bmod 4)$, то $c-d \equiv d+1(\bmod 2)$. Поэтому из $(21)$ получаем

$$
\left(\frac{a}{p}\right)= \begin{cases}(-1)^{d+1}, & \text { если одновременно } a \equiv 2,3(\bmod 4) \text { и } t \equiv 1,2(\bmod 4), \\ (-1)^{d} & \text { в остальных случаях. }\end{cases}
$$

Теорема доказана. 
СлЕДСТвИЕ. Обозначим

$$
d^{(1)}=\left|P_{a}^{([a / 2])}\left(a,-n_{1}\right) \cap I\right|,
$$

где I - отрезок из (17). Тогда

$$
\left(\frac{a}{p}\right)= \begin{cases}(-1)^{d^{(1)}+1}, & \text { если одновременно } a \equiv 2,3(\bmod 4) u t \equiv 1,2(\bmod 4), \\ (-1)^{d^{(1)}} & \text { в остальных случаях. }\end{cases}
$$

Утверждение получается с использованием леммы.

Отметим, что доказанная теорема представляет собой некоторую комбинаторную интерпретацию леммы Гаусса. Следствие из нее позволяет предположить, что формулы (22), (23) могут быть развернуты в рекуррентную процедуру для быстрого вычисления символа Якоби, параллельную известной процедуре вычисления, основанной на квадратичном законе взаимности (см. [6, гл. II]); обе они напоминают известньй алгоритм Евклида. Заметим, что доказательство теоремы 1 не использует закона взаимности и носит чисто комбинаторньй характер. Фактическое вычисление символа Лежандра с использованием формулы (23), очевидно, не медленнее обычной процедуры.

Применим теперь теорему 1 к описанию в явном виде множества всех простых чисел $p$, принадлежаших некоторому заданному конечному множеству, при условии $\left(\frac{a}{p}\right)=1$ для некоторого целого числа $a$.

Введем дополнительно несколько обозначений. Определим функцию $\pi$ на целых чис$\operatorname{лаx~} z$ :

$$
\pi(z)= \begin{cases}0, & \text { если } z \text { четно, } \\ 1, & \text { если } z \text { нечетно. }\end{cases}
$$

Тогда в стандартном представлении нечетного простого $p$ через целое $a, 0<a<p$, имеем

$$
\begin{array}{rlrl}
\pi(t)+\pi\left(n_{1}\right) & =1, & & \text { если } a \text { нечетно, } \\
\pi\left(n_{1}\right) & =1, & \text { если } a \text { четно. }
\end{array}
$$

Через $Z_{a}^{*}$ обозначаем множество всех целых чисел, меньших натурального числа $a$ и взаимно простых с ним, $\left|Z_{a}^{*}\right|=\varphi(a), \varphi$ - функция Эйлера.

Так как мы хотим рассматривать условие $\left(\frac{a}{p}\right)=1$, можно было бы предположить, что $a$ свободно от квадратов. Однако на практике установление этого свойства для достаточно больших $a$ требует немалого времени. Поэтому с целью наибольшей эффективности предлагаемого способа описания простых чисел $p$ c $\left(\frac{a}{p}\right)=1$ мы предлагаем далее лишь вьполнение свойства $4 \nmid a$. Обозначим

$$
\widetilde{a}= \begin{cases}2 a, & a \equiv 1(\bmod 4) \\ 4 a, & a \equiv 2,3(\bmod 4) .\end{cases}
$$

Через $P_{r}(a)$ для $r \in Z_{\widetilde{a}}^{*}$ обозначаем множество (бесконечное) всех простых чисел $p, \mathrm{y}$ которых в представлении $(2)$ через $\widetilde{a}$ имеем $n_{1}=r$, т.е. $p \equiv r(\bmod \widetilde{a})$. 
Теорема 2. Пусть $p$ - простое, $a-$ иелое число, $4 \nmid a, 0<a<p$. Тогда условие

$$
\left(\frac{a}{p}\right)=1
$$

равносильно свойству

$$
p \in \bigcup_{r \in R(a)} P_{r}(a),
$$

где $R(a) \subset Z_{a}^{*}$ и описывается следуюшим образом:

1) если $a \equiv 1(\bmod 4)$, mo

$$
R(a)=\left\{a\left(1-\pi\left(n_{1}\right)\right)+n_{1}: n_{1} \in Z_{a}^{*},\left(\frac{n_{1}}{a}\right)=1\right\}, \quad|R(a)|=\frac{1}{2} \varphi(a) ;
$$

2) ecлu $a \equiv 3(\bmod 4)$, mo

$$
\begin{gathered}
R(a)=\left\{a \delta\left(n_{1}, d\right)+n_{1}: n_{1} \in Z_{a}^{*}\right\}, \quad|R(a)|=\varphi(a), \\
\delta\left(n_{1}, d\right)= \begin{cases}0, & \text { если } \pi\left(n_{1}\right)=1, \pi(d)=0, \\
1, & \text { если } \pi\left(n_{1}\right)=0, \pi(d)=1, \\
2, & \text { если } \pi\left(n_{1}\right)=1, \pi(d)=1, \\
3, & \text { если } \pi\left(n_{1}\right)=0, \pi(d)=0 ;\end{cases}
\end{gathered}
$$

3) $е с л u a \equiv 2(\bmod 4)$, mo

$$
\begin{aligned}
R(a) & =R_{0}(a) \cup R_{1}(a), \quad|R(a)|=2 \varphi(a), \\
R_{0}(a) & =\left\{a \delta(t, d)+n_{1}: n_{1} \in Z_{a}^{*}, \pi(t)=0\right\}, \\
R_{1}(a) & =\left\{a \delta(t, d)+n_{1}: n_{1} \in Z_{a}^{*}, \pi(t)=1\right\},
\end{aligned}
$$

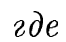

$$
\delta(t, d)= \begin{cases}0, & \text { если } \pi(t)=0, \pi(d)=0 \\ 1, & \text { если } \pi(t)=1, \pi(d)=1 \\ 2, & \text { если } \pi(t)=0, \pi(d)=1 \\ 3, & \text { если } \pi(t)=1, \pi(d)=0\end{cases}
$$

здесь $\pi$ - функиия четности из (24).

ДокАЗАТЕльСтво. Предполагаем вначале, что $\left(\frac{a}{p}\right)=1$, и покажем, что тогда для $p$ вьполнено (26). Рассматриваем стандартное представление $p=a t+n_{1}, n_{1}<a$. Докажем утверждение для $a$ из пункта 2$)$. Итак, пусть $a \equiv 3(\bmod 4)$. Тогда $\widetilde{a}=4 a$.

a) Пусть сначала $n_{1}$ - какое-либо нечетное число в $Z_{a}^{*}$. Тогда $t$ четно (см. (25)). Находим $n_{2}$, отрезок $I$ и число $d$. Покажем, что $\pi(d)=0$ равносильно $t \equiv 0(\bmod 4)$. Действительно, пусть $\pi(d)=0$, и предположим от противного, что $t \equiv 2(\bmod 4)$.

Тогда согласно теореме 1

$$
\left(\frac{a}{p}\right)=(-1)^{d+1}=-1,
$$


что противоречит предположению. Непосредственно по теореме 1 получаем и обратное утверждение: если $t \equiv 0(\bmod 4)$, то $\pi(d)=0$. Полагаем $t=4 t_{1}$. Тогда $p=4 a t_{1}+n_{1}$, т.е. в рассматриваемом случае

$$
\delta\left(n_{1}, d\right)=0, \quad r \in R(a)=\left\{0 \cdot a+n_{1}, n_{1} \in Z_{a}^{*} \text { - нечетное число }\right\} .
$$

Аналогично доказьвается, что $\pi(d)=1 \Longleftrightarrow t \equiv 2(\bmod 4)$, или $t=4 t_{1}+2$. Отсюда получаем

$$
\delta\left(n_{1}, d\right)=2, \quad r \in R(a)=\left\{2 a+n_{1}, n_{1} \in Z_{a}^{*} \text { - нечетное число }\right\} .
$$

б) Пусть теперь $n_{1}-$ какое-либо четное число в $Z_{a}^{*}$. Аналогично предыдущему, используя теорему 1 , находим, что $\pi(d)=0 \Longleftrightarrow t \equiv 3(\bmod 4)$, a $\pi(d)=1 \Longleftrightarrow t \equiv 1$ $(\bmod 4)$. Отсюда в первом случае получаем, что

$$
\delta\left(n_{1}, d\right)=3, \quad r \in R(a)=\left\{3 a+n_{1}, n_{1} \in Z_{a}^{*} \text {-четное число }\right\},
$$

а во втором

$$
\delta\left(n_{1}, d\right)=1, \quad r \in R(a)=\left\{a+n_{1}, n_{1} \in Z_{a}^{*} \text { - четное число }\right\} .
$$

Таким образом, мы показали, что при $a \equiv 3(\bmod 4)$ из $\left(\frac{a}{p}\right)=1$ следует, что для $p$ выполнено условие $(26)$. Для $a \equiv 1$ и $a \equiv 2(\bmod 4)$ тот же факт устанавливается совершенно аналогично.

Непосредственной проверкой по формуле из теоремы 1 получаем также обратное утверждение: если для простого числа $p$ выполнено условие $(26)$, то $\left(\frac{a}{p}\right)=1$. Теорема доказана.

Теорему 2 удобно применять для эффективного нахождения всех простых модулей $p$, принадлежащих некоторому заданному конечному множеству $M$, при условии, что данная конечная последовательность целых чисел $a_{1}, a_{2}, \ldots, a_{l}$ имеет свойства

$$
\left(\frac{a_{i}}{p}\right)=1, \quad i=1,2, \ldots, l,
$$

для каждого $p \in M$.

Отметим в заключение, что полученные результаты, по-видимому, обобщаются на случай произвольного нечетного $b$ и символа Якоби $\left(\frac{a}{p}\right) ; a$ взаимно просто с $b$.

\section{СПИСОК ЦИТИРОВАННОЙ ЛИТЕРАТУРЫ}

[1] Коблиц Н. Курс теории чисел и криптографии. М.: ТВП, 2001.

[2] Brands S.A., Gill R.D. Cryptography, statistics, and pseudorandomness. I // Probab. Math. Statist. 1995. V. 15. P. 101-114.

[3] Brands S. A., Gill R. D. Cryptography, statistics, and pseudorandomness. II // Probab. Math. Statist. 1995. V. 16. P. 1-17.

[4] Anchel M., Goldfeld D. Zeta functions, one-way functions, and pseudorandom number generators // Duke Math. J. 1997. V. 88. P. 371-390.

[5] Боревич З. И., Шафаревич И. Р. Теория чисел. М.: Наука, 1964.

[6] Хассе Г. Лекции по теории чисел. М.: ИЛ, 1953. 